\title{
Association of Lipoprotein and Thyroid Hormones With Cognitive Dysfunction in Patients With Systemic Lupus Erythematosus
}

Li Lu

The State Key Laboratory of Pharmaceutical Biotechnology, Division of Immunology, Medical School, Nanjing University

\section{Wei Kong}

Department of Rheumatology and Immunology, Nanjing Drum Tower Hospital, The Affiliated Hospital of Nanjing University Medical School

\section{Kangxing Zhou}

Department of Rheumatology and Immunology, Nanjing Drum Tower Hospital, The Affiliated Hospital of Nanjing University Medical School

\section{Jinglei Chen}

The State Key Laboratory of Pharmaceutical Biotechnology, Division of Immunology, Medical School, Nanjing University

\section{Yayi Hou}

The State Key Laboratory of Pharmaceutical Biotechnology, Division of Immunology, Medical School, Nanjing University

\section{Huan Dou ( $\square$ douhuan@nju.edu.cn )}

The State Key Laboratory of Pharmaceutical Biotechnology, Division of Immunology, Medical School, Nanjing University

Jun Liang

Department of Rheumatology and Immunology, Nanjing Drum Tower Hospital, The Affiliated Hospital of Nanjing University Medical School

\section{Research Article}

Keywords: Neuropsychiatric systemic lupus erythematosus (NPSLE), Cognitive dysfunction, thyroid hormones (T3, T4), Lipoprotein, Correlation analysis

Posted Date: December 7th, 2020

DOI: https://doi.org/10.21203/rs.3.rs-115002/v1 
License: (c) (i) This work is licensed under a Creative Commons Attribution 4.0 International License. Read Full License

Version of Record: A version of this preprint was published at BMC Rheumatology on June 9th, 2021. See the published version at https://doi.org/10.1186/s41927-021-00190-7. 


\section{Abstract}

Background: Neuropsychiatric systemic lupus erythematosus (NPSLE) occurs up to $75 \%$ of adult SLE patients, and is one of the major causes of death in SLE patients. Cognitive dysfunction is a typical clinical feature of NPSLE, which seriously affects the quality of life of patients. Dyslipidemia and thyroid disease, which were prevalent in SLE patients, both related to the neuropsychiatric disturbances, including significant psychiatric and cognitive disturbance. This study aimed to investigate whether cognitive dysfunction in patients with systemic lupus erythematosus (SLE) was related to the expression of serum thyroid hormone and lipoproteins.

Methods: A total of 121 patients with SLE and 65 healthy controls (HCs) at Nanjing Drum Tower Hospital completed a cognitive function test, then 81 SLE patients were divided into high cognition $(n=33)$ group and low cognition group $(n=48)$. The differences in clinical and laboratory tests and the correlations between IgG, IgM, albumin, T3, and T4 levels and cognitive function were analyzed. The enzyme-linked immunosorbent assay was used to determine the serum levels of 4 lipoproteins (APOE, APOA1, IGF-1, and IGFBP7) in 81 patients.

Results: Patients with SLE were less educated with abnormal cognitive function compared with HCs. The levels of albumin, T3 $(\mathrm{P}<0.05)$, and T4 decreased in low-cognition patients, while $\mathrm{D}$-dimer, anti-dsDNA antibody, and IgM levels increased. The serum IgG and IgM levels showed a significant negative correlation with partial cognitive function. The serum T3 and T4 levels positively correlated with cognition. The expression of APOE, APOA1, IGF-1, and IGFBP7 showed no difference between the highand low-cognition groups. However, the serum APOE level negatively correlated with Line Orientation, APOA1 positively correlated with Coding, and IGFBP7 negatively correlated with Graphic copy $(P<0.05)$, and IGF-1 had no correlation with any cognitive functions.

Conclusions: Thyroid hormones (T3 and T4) and lipoproteins (APOE, APOA1, and IGFBP7) were associated with cognitive dysfunction in SLE. Whether T3 and T4 can be used in clinical practice as the biomarkers of cognitive dysfunction in SLE needs further exploration.

\section{Introduction}

Systemic lupus erythematosus (SLE) is a chronic systemic autoimmune disease characterized by autoantibody production and immune complex deposition, culminating in destructive tissue injury to multiple organ systems including the central nervous system (CNS) [1-2]. Neuropsychiatric SLE (NPSLE) is a form of SLE associated with severe neuropsychiatric (NP) syndromes, including various neurological and psychiatric features [3]. Several studies estimated that up to $75 \%$ of patients with SLE experienced NPSLE [4-5]. The symptoms of NPSLE include mood disorders, confusion, headache, and cognitive dysfunction, which significantly degrades the quality of life and affects the survival of patients [6]. However, the underlying disease mechanisms remain largely unknown, as neuropsychiatric symptoms are nonspecific, clinically validated biomarkers for diagnosis are nonexistent, and NPSLE diagnosis is 
difficult, often leading to palliative rather than therapeutic protocols [7-8]. Cognitive function assessment is one of the valuable clinical skills used to screen for cognitive impairment and assess the severity of the disease [9]. Many cognitive function assessment tools are available, including Taiwan Mental State Examination [10], Montreal Cognitive Assessment [11], AD8 Dementia Screening Interview [12], and so forth. However, the assessment of cognitive function is incomplete and insensitive to mild cognitive impairment due to the relative simplicity of these scales. Although a complete set of neuropsychological tests can comprehensively assess the level of cognitive function and the severity of the cognitive impairment, it generally takes a long time, is prone to subject fatigue, and is especially not suitable for the clinical evaluation of the elderly [13]. Therefore, tools that are simple to implement and can relatively comprehensively evaluate cognitive function have a great application value. The Repeatable Battery for the Assessment of Neuropsychological Status (RBANS) overcomes the aforementioned shortcomings. It was originally used to identify the pathological decline of cognitive function in the elderly and also could screen the neuropsychological function status of the general population [14]. Later studies applied it to bipolar disorder [15], multiple sclerosis [16], cerebrovascular disease [17], epilepsy [18], and other diseases with cognitive impairment in different fields. The results showed that RBANS could be used as an effective neuropsychological function screening tool, with good internal consistency, test-retest reliability, structural validity, and parallel validity, and sex has no significant effect on the evaluation results of RBANS. These findings showed that RBANS could be a favorable means of assessing cognitive function in patients with SLE.

Dyslipidemia is prevalent in patients with SLE with an incidence ranging up to $75 \%$. It is characterized as disordered low-density lipoprotein ( $L D L)$ and/or decreased high-density lipoprotein (HDL) levels in the serum [19]. Dyslipidemia is associated with disease activity, such as kidney damage and cardiovascular disease, which are the most common complications in patients with SLE and closely related to the longterm prognosis [20-21]. In addition, dyslipidemia is believed to be closely associated with the occurrence of cognitive dysfunction and Alzheimer's disease (AD). The hippocampus is critically important for learning and memory. Researchers found that high-fat diet-induced rats had impaired neurogenesis in the dentate gyrus of the hippocampus [22], decreased hippocampal production of brain-derived neurotrophic factor [23], increased apoptosis of hippocampal neurons, and an associated decrease in the weight of the hippocampus[24], which likely plays a key role in neuronal loss, leading to learning and memory deficits. Nonthyroidal illness syndrome is also prevalent in SLE and characterized by decreased serum triiodothyronine (T3), thyroxine (T4), and thyroid-stimulating hormone levels [25-27]. Thyroid hormones can influence all aspects of lipid metabolism including synthesis, mobilization, and degradation, thus resulting in dyslipidemia [28]. Earliest descriptions of thyroid disease showed a link with neuropsychiatric disturbances, including significant psychiatric and cognitive disturbance, classical slowness of thought, and increased depressive symptoms [29]. Treatment with T4 helps improve the disturbance in mood [30], but not neurocognitive function, especially complex attention tasks and verbal memory tests [31]. Inextricable links exist between SLE, dyslipidemia, and abnormal thyroid hormone levels. However, whether dyslipidemia and thyroid hormone abnormalities in patients with lupus are related to cognitive dysfunction remains unknown. 
In this study, the correlation between cognitive function and the levels of serum thyroid hormones ( $\mathrm{T} 3$ and T4) and lipoproteins (APOE, APOA1, and IGFBP7) in SLE were evaluated. This study aimed to discover the changes in the levels of serum biomarkers and proteins possibly related to the cognitive function in SLE, thus prompting diagnosis or predicting prognosis of SLE.

\section{Materials And Methods}

\subsection{Patients and study design}

A total of 121 patients with SLE who visited the Department of Rheumatology, Nanjing Drum Tower Hospital, Nanjing, China, from May 2019 to May 2020 were prospectively enrolled. All patients were diagnosed according to the SLE criteria of the American College of Rheumatology [32]. The disease activity of these patients was measured using the Systemic Lupus Erythematosus Disease Activity Index (SLEDAI) [33-34]. Patients who had other autoimmune diseases; had a history of familial hyperlipidemia and/or thyroid disease, diabetes mellitus, and/or other rheumatic diseases; and took lipid-lowering agents or thyroid medications were excluded. Active patients were classified into two groups: low-cognition group ( $n=33$; RBANS score: $51-90$ ) and high-cognition group ( $n=48$; RBANS score: $91-130)$, based on the RBANS score. Patients who developed neuropsychiatric syndromes not attributable to SLE (electrolyte imbalances, infections, or medications) were excluded. Meanwhile, 65 age- and gender-matched healthy controls (HCs) were recruited from the Physical Examination Center of Nanjing Drum Tower Hospital. This study was approved by the ethics committee at The Affiliated Drum Tower Hospital of Nanjing University Medical School (ID: SC201700201) and undertaken according to the guidelines of the Declaration of Helsinki. At entry, patients completed a standardized medical history, laboratory tests, and analyses. All the detections were carried out at the clinical laboratory of Nanjing Drum Tower Hospital. The demographic features of the patients and HC samples are shown in Table 1.

\subsection{Cognitive dysfunction study}

The cognitive dysfunction in SLE is characterized by deficits in attention, learning and recall, verbal and nonverbal fluency, language, visuospatial skills, executive functions, and motor dexterity [35-37]. A total of 121 patients with NPSLE and 65 normal volunteers were invited to participate in this study. All participants provided additional medical information using an ad-hoc questionnaire and had a physical examination.

The RBANS consisted of five indexes: immediate memory, visuospatial/constructional, attention, language, and delayed memory [38]. Stimuli were contained in a wire-bound, easel-type booklet, making the test easily portable and allowing for bedside administration. The total administration time was 20-30 min. The battery indexes were assessed using the following tests [38]:

a. List Learning: The examiner read aloud a list of 10 words, which were semantically unrelated, early acquired, relatively high-imagery, and as phonetically unique as possible. The participants were asked to recall (without regard for order) as many of these items as possible. After four trials, the 
number of words correctly recalled on the fourth trial was used as a measure of verbal learning and memory; the total score was 40 points.

b. Immediate Story Memory: The examiner read a short story including 12 keywords aloud twice, and then the participants were asked to recall this story as possible. The number of keywords correctly recalled was used as a measure of immediate story memory to avoid complicated scoring rules; the total score was 24 points.

c. Figure Copy: The participants were asked to remember a geometric figure comprising 10 parts and then copy this figure in 4 min. Each part received a 2-point score (accuracy and placement); the total score was 20 points.

d. Line Orientation: Two lines were presented at an angle, and the participants needed to select the corresponding lines on a simultaneously presented array. The subject's task on each item was to identify the matching lines. One point was given for each correctly matched line; the total score was 20 points.

e. Picture Naming: The participants were shown 10 pictures and asked to name the objects of the pictures. Semantic cues were given if the object was obviously misperceived (e.g., "umbrella" for mushroom). Naming correctly (including the correct answer according to the prompts) was used as a measure of picture naming; the total score was 10 points.

f. Semantic Fluency: The participants should generate the words as much as possible for a given semantic category (e.g., fruits and vegetables) within $60 \mathrm{~s}$. The semantic categories aimed to minimize retrieval demands and thereby more specifically tap semantic stores rather than retrieval strategies. One point was given for each correct word; the total score was 20 points.

g. Digit Span: The Digit Span was analogous to digits forward on the Wechsler Adult Intelligence Scale (WAIS). There were two string of digits in each item, increasing from 2 to 9 digits. The second given length string was read when the first string was failed. The failure of attempts of two same items was given 0 points; the total score was 16 points.

h. Coding: First, the participants were provided with a row of graphics, each of which corresponded to a number. Later, they were provided with some figures without numbers. They were asked to fill in the number under each figure provided for the second time as fast as possible, according to the corresponding relationship between the figure and the number provided for the first time. Numbers rather than symbols were chosen for the response to avoid the possible detrimental effect of constructional apraxia on performance. The score was the total number of items completed in $90 \mathrm{~s}$.

i. List Recall: In this test, the participants were asked to recall the words provided in the "List Learning" test as many as possible. One point was given for each correctly recalled words, and the total score was 16 points.

j. List Recognition: In this test, the examiner read 20 words aloud, including 10 words provided in the "List Learning" test. The participants were asked to answer whether the words were read before. One point was given for each correct recognition, and the total score was 20 points. 
k. Delay Story Recall: In this test, the participants were asked to recall the story provided in the "Story Memory" test. The number of keywords correctly recalled was used as a measure of delay story memory to avoid complicated scoring rules. One point was given for each correctly recalled keywords, and the total score was 12 points.

I. Figure Recall: In this test, the participants were asked to recall and draw the figure provided in the "Figure Copy" test as accurate as possible. Each part received a 2-point score (accuracy and placement) for 20 possible points.

SCALING: For the purpose of the present study, a single reference sample was used to provide the scaling metrics. The norm was established based on the test results of 540 people aged $20-89$ years. Raw scores were converted into scaled scores for all participants in the present study using the same scaling metric, regardless of age [38-39].

\subsection{Measurement of serum autoantibodies}

The levels of autoantibodies and lipoproteins of patients in the serum were measured within 1 month of the cognitive function testing. In this phase of the study, participants had a blood sample drawn. A venous puncture was done, and $2 \mathrm{~mL}$ of blood was collected in a serum separator tube. The serum from the patients was prepared by centrifugation at $3000 \mathrm{rpm}$ for $10 \mathrm{~min}$ in a clinical centrifuge. The autoantibodies, including anti-ds-DNA antibody, anti-Sm antibody, anti- Ribonucleoprotein antibody (antiRNP), anti-Sjögren's syndrome A antibody (anti-SSA), anti- Sjögren's syndrome B antibody (anti-SSB), anti-ribosomal-P antibody, and anti- $\beta 2$-glycoprotein-I antibody, and immunoglobulins including Immunoglobulin G (IgG), Immunoglobulin M (IgG), Immunoglobulin A (IgA), and Immunoglobulin E (IgE), were analyzed.

\subsection{Enzyme-linked immunosorbent assay}

The expression of apolipoprotein E (APOE, RayBio, USA), apolipoprotein A1 (APOA1, R\&D system, USA), insulin-like growth factor-1 (IGF-1, Fcmacs, China), and insulin-like growth factor binding protein 7 (IGFBP7, Arigobio, China) were detected using an enzyme-linked immunosorbent assay (ELISA) kit following the manufacturer's protocols. In short, a 96-well plate was coated with the antibody (100 $\mu \mathrm{L} /$ well) in duplicate and incubated at $4{ }^{\circ} \mathrm{C}$ overnight. The plate was then washed five times with wash buffer and dried gently on a lint-free paper towel. After the sample was diluted, $100 \mu \mathrm{L}$ of the detection samples was added to each well, and the plate was incubated for $2 \mathrm{~h}$ at room temperature. An equal volume $(100 \mu \mathrm{L} /$ well $)$ of the substrate solution was added, incubated at room temperature for $30 \mathrm{~min}$, and then protected from light. The reaction was stopped by adding $100 \mu \mathrm{L} /$ well of $1 \mathrm{M} \mathrm{H} 2 \mathrm{SO}$, and the optical density was determined at a wavelength of $450 \mathrm{~nm} 5 \mathrm{~min}$ later [40]. The results were fitted to the standard curve, and the detection ranges were as follows: APOE $(1.5-400 \mathrm{ng} / \mathrm{mL})$, APOA1 $(6.3-200$ $\mathrm{ng} / \mathrm{mL})$, IGF-1 (62.5-4000 pg/mL), and IGFBP7 (625-40000 pg/mL).

\subsection{Statistical analysis}


Data were expressed as means \pm standard deviation. Differences between two groups were determined using the unpaired-sample Student $t$ test if the variance was normally distributed, and the Mann-Whitney $U$ test was used for non-normally distributed data. Differences among observed frequencies were tested using the chi-square test, while Pearson's correlation coefficient was used to calculate the correlation between variables [40]. The correlations were analyzed using Spearman's correlation test. Data were analyzed and visualized using SPSS16.0 software or GraphPad Prism 7.0 (GraphPad Software, CA, USA), and a two-tailed $P$ value $<0.05$ was considered statistically significant.

\section{Results}

\subsection{Clinical characteristics and cognitive function of patients with SLE and HCs}

The demographic features of 121 patients with SLE and $65 \mathrm{HCs}$ in the study are summarized in Table 1. The mean age of patients and HCs was $33.88 \pm 11.77$ and $36.69 \pm 13.88$ years, respectively. The mean level of education was $11.95 \pm 3.05$ years in the patient group, which was significantly lower than that in the HC group $(P<0.01)$.

Table 1. Demographic features of the 121 SLE patients and 65 healthy controls in the study.

\begin{tabular}{|llll|}
\hline Variables & SLE $(\mathrm{n}=121)$ & $\mathrm{HC}(\mathrm{n}=65)$ & $P$ Value \\
\hline Age, (years) & $33.88( \pm 11.77)$ & $36.69( \pm 13.88)$ & 0.150 \\
\hline Females, $\mathrm{n}(\%)$ & $106(87.60 \%)$ & $50(76.92 \%)$ & 0.093 \\
\hline Education, (years) & $11.95( \pm 3.05)$ & $13.28( \pm 4.58)$ & $<0.05^{\star}$ \\
\hline SLEDAl & $10(0-47)$ & $\mathrm{NA}$ & $/$ \\
\hline
\end{tabular}

Data are expressed as median (minimum - maximum, number (percentage), or mean \pm standard deviation (SD) values. $P$ values are based on independent sample t-test and Chi-square test for normally distributed variables. ${ }^{\star} P<0.05$, ${ }^{\star \star} P<0.01$, ${ }^{\star \star \star} P<0.005$, vs. Low RBANS Score. The Graph Pad Prism 7.0 and SPSS Statistics 16.0 software were used for statistical analysis.

Afterward, an RBANS was performed on 121 patients and $65 \mathrm{HCs}$. Significant differences were found in List Learning $(P<0.0001)$ between patients with SLE and HCs, while Immediate Story Recall, Picture Naming, Coding, List Recall, List Recognition Test, and Delayed Story Recall (all $P<0.01$ ), as well as Judgment of Line Orientation, Digit Span, and Figure Recall (all $P<0.05$ ), were lower in patients with SLE. However, Figure Copy and Semantic Fluency showed no differences (Table 2). These tests corresponded to five cognitive domains: immediate memory, visuospatial/construction, attention, language, and delayed memory. After categorizing the aforementioned cognitive function tests according to these five cognitive domains, the difference between patients and normal volunteers was analyzed. The results showed that levels of immediate memory, visuospatial/construction, attention, and delayed memory were significantly lower in patients than in HCs, while language features showed no obvious difference (Fig. 1). 
Table 2. The data of commonly cognitive assessment.

\begin{tabular}{|c|c|c|c|}
\hline RBANS score & SLE $(n=121)$ & $\mathrm{HC}(\mathrm{n}=65)$ & $P$ Value \\
\hline \multicolumn{4}{|l|}{ Immediate memory } \\
\hline List Learning, median (range) & $24(5-39)$ & $29(15-36)$ & $<0.005^{\star \star \star}$ \\
\hline Immediate Story Memory, median (range) & $13(1-22)$ & $16(1-21)$ & $<0.01$ ** \\
\hline \multicolumn{4}{|l|}{ Visuospatial/constructional } \\
\hline Figure Copy, median (range) & $20(14-20)$ & $20(17-20)$ & 0.2963 \\
\hline Line Orientation, median (range) & $17(8-20)$ & $18(9-20)$ & $<0.05^{\star}$ \\
\hline \multicolumn{4}{|l|}{ Language } \\
\hline Picture Naming, median (range) & $9(7-10)$ & $9(8-10)$ & $<0.01 * *$ \\
\hline Semantic Fluency, median (range) & $21(7-33)$ & $23(11-35)$ & 0.0873 \\
\hline \multicolumn{4}{|l|}{ Attention } \\
\hline Digit Span, median (range) & $13(6-16)$ & $14(8-16)$ & $<0.05^{\star}$ \\
\hline Coding, median (range) & $46(4-70)$ & $54(13-74)$ & $<0.01^{\star \star}$ \\
\hline \multicolumn{4}{|l|}{ Delayed memory } \\
\hline List Recall, median (range) & $5(0-10)$ & $7(2-10)$ & $<0.01 * \star$ \\
\hline List Recognition, median (range) & $20(16-20)$ & $20(17-20)$ & $<0.01$ ** \\
\hline Delay Story Recall, median (range) & $7(0-12)$ & $9(0-12)$ & $<0.01^{\star \star}$ \\
\hline Figure Recall, median (range) & $15(5-20)$ & $16(7-20)$ & $<0.05^{\star}$ \\
\hline
\end{tabular}

Data are expressed as mean \pm standard deviation (SD) values. $P$ values are based on independent

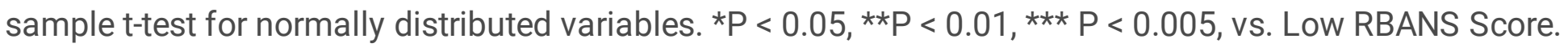
The Graph Pad Prism 7.0 software were used for statistical analysis.

\subsection{Demographic features, clinical manifestations, and treatment of patients}

Based on the RBANS score, 81 patients were divided into high-cognition group ( $n=33)$ and low-cognition group $(n=48)$. As shown in Table 3 , no statistically significant differences in age and sex were found between the two groups. The mean level of education was $10.77 \pm 3.64$ years in the low-cognition group, which was lower than that in the high-cognition group $(P<0.01)$. The levels of SLEDAI $(13.91 \pm 11.79 \mathrm{vs}$ $9.38 \pm 5.79)$ significantly increased in patients with low cognition compared with those in high cognition (all $P<0.05$ ).

Table 3. Demographic features, clinical manifestations, and treatment of SLE patients. 


\begin{tabular}{|c|c|c|c|}
\hline SLE characteristics & Low Cognition $(n=48)$ & High Cognition $(n=33)$ & $P$ Value \\
\hline \multicolumn{4}{|l|}{ Demographic } \\
\hline Age, (years) & $34.67( \pm 12.03)$ & $32.23( \pm 10.98)$ & 0.3530 \\
\hline Females, n (\%) & $40(83.33 \%)$ & $30(90.90 \%)$ & 0.763 \\
\hline Education, (years) & $10.77( \pm 3.64)$ & $13.26( \pm 3.21)$ & $<0.01^{\star \star}$ \\
\hline SLEDAI, mean $\pm(S D)$ & $13.91( \pm 11.79)$ & $9.38( \pm 5.79)$ & $<0.05^{\star}$ \\
\hline \multicolumn{4}{|l|}{ Clinical chart review (\%) } \\
\hline Rash, (\%) & $10(21.28 \%)$ & $6(18.18 \%)$ & 0.768 \\
\hline Mucosal ulcers, $(\%)$ & $2(4.26 \%)$ & $1(3.03 \%)$ & 1.000 \\
\hline Hematuria, (\%) & $22(46.81 \%)$ & $13(39.33 \%)$ & 0.510 \\
\hline Proteinuria, (\%) & $22(46.81 \%)$ & $13(39.33 \%)$ & 0.510 \\
\hline Pyuria, (\%) & $14(39.79 \%)$ & $11(33.33 \%)$ & 0.736 \\
\hline Arthritis, (\%) & $8(17.02 \%)$ & $5(15.15 \%)$ & 0.823 \\
\hline Vasculitis, (\%) & $4(8.51 \%)$ & $0(0 \%)$ & 0.231 \\
\hline Pleurisy, (\%) & $1(2.13 \%)$ & $1(3.03 \%)$ & 1.00 \\
\hline Pericarditis, (\%) & $1(2.13 \%)$ & $0(\%)$ & 1.00 \\
\hline Low complement, (\%) & $36(76.60 \%)$ & $27(81.82 \%)$ & 0.574 \\
\hline Anemia, (\%) & $11(23.40 \%)$ & $1(3.03 \%)$ & $<0.05^{\star}$ \\
\hline Thrombocytopenia, (\%) & $11(23.40 \%)$ & $4(12.12$ & 0.203 \\
\hline Leukopenia, (\%) & $11(23.40 \%)$ & $7(21.21 \%)$ & 0.817 \\
\hline Lupus nephritis, (\%) & $19(40.43 \%$ & $20(60.61 \%)$ & 0.075 \\
\hline Neurological disorder, (\%) & $6(12.77 \%)$ & $5(15.15 \%)$ & 1.00 \\
\hline \multicolumn{4}{|l|}{ Current medication (\%) } \\
\hline Prednisone (\%) & $31(64.58 \%)$ & $18(54.54 \%)$ & 0.425 \\
\hline Hydroxychloroquine (\%) & $29(61.70 \%)$ & $19(57.58 \%)$ & 0.711 \\
\hline Cyclophosphamide (\%) & $5(10.64 \%)$ & $3(9.09 \%)$ & 1.000 \\
\hline Azathioprine (\%) & $1(2.13 \%)$ & $1(3.03 \%)$ & 1.000 \\
\hline Methotrexate (\%) & $2(4.26 \%)$ & $2(6.06 \%)$ & 1.000 \\
\hline Cyclosporine (\%) & $0(0 \%)$ & $2(6.06 \%)$ & 0.326 \\
\hline
\end{tabular}


Data are expressed as number (percentage). $P$ values are based on Chi-square test for normally distributed variables. ${ }^{\star} \mathrm{P}<0.05,{ }^{\star} * \mathrm{P}<0.01,{ }^{\star * \star} \mathrm{P}<0.005$, vs. Low RBANS Score. The SPSS Statistics 16.0 software were used for statistical analysis.

Patients with low cognition had more frequent anemia (23.4\% vs $3.03 \%$ ) compared with patients with high cognition $(P<0.05)$. Meanwhile, the prevalence of rash, mucosal ulcers, hematuria, proteinuria, pyuria, arthritis, vasculitis, pleurisy, pericarditis, low complement, thrombocytopenia, leukopenia, lupus nephritis, and neurological disorder showed no difference between the two groups. In addition, the treatment with prednisone, hydroxychloroquine, cyclophosphamide, azathioprine, methotrexate, and cyclosporine was not different between the two groups (Table 3 ).

\subsection{Comparison of clinical and laboratory characteristics of patients}

The study investigated the laboratory parameters of patients enrolled in this study (Table 4). Patients with low cognition had substantially lower albumin $(31.76 \pm 5.79 \mathrm{mg} / \mathrm{dL}$ vs $34.91 \pm 4.13 \mathrm{mg} / \mathrm{dL} ; P<$ $0.01)$, T3 $(3.20 \pm 1.03 \mathrm{ng} / \mathrm{dL}$ vs $3.71 \pm 1.06 \mathrm{ng} / \mathrm{dL} ; P<0.05)$, and T4 levels $(12.51 \pm 3.43 \mu \mathrm{g} / \mathrm{dL}$ vs $14.97 \pm$ $2.14 \mu \mathrm{g} / \mathrm{dL} ; P<0.05)$. The levels of anti-dsDNA antibody $(P<0.05)$ and $\operatorname{lgM}(P<0.01)$ and the number of B cells $(P<0.05)$ were higher and the D-dimer levels were considerably higher in patients with low cognition $(P<0.001)$. The incidence of other clinical indexes, including globulin, 24-h urine protein (24-h UP), C3, C4, C-reactive protein, and creatinine ( $\mathrm{Cr}$ ), displayed no difference between the two groups.

In addition, the difference in the proportion of various blood cells in the peripheral blood between the two groups was detected. The results showed that the number of $B$ cells was higher in the low-cognition group $(0.15 \pm 0.16 \%$ vs $0.10 \pm 0.13 \% ; P<0.05)$, but no difference was found in the numbers of erythrocytes, WBCs, $\mathrm{CD} 3^{+}$cells, $\mathrm{CD} 3^{+} \mathrm{CD} 4^{+}$cells, $\mathrm{CD} 3^{+} \mathrm{CD} 8^{+}$cells, and natural killer cells between the groups (Table 4). As shown in Table 4, the serum levels of anti-dsDNA antibodies $(P<0.05)$ and $\operatorname{lgM}(P<$ 0.01 ) were both higher in patients with low cognition. However, no statistically significant differences in positive rates of anti-Sm antibodies, anti-RNP antibodies, anti-SSA antibodies, anti-SSB antibodies, antiRib $P$ antibodies, and anti- $\beta_{2}$ GPI antibodies, as well as the levels of $\lg G$, $\lg A$, and $\lg E$, were found between the two groups.

Table 4. Comparison of clinical and laboratory characteristics of SLE patients. 


\begin{tabular}{|c|c|c|c|}
\hline SLE characteristics & Low Cognition $(n=48)$ & High Cognition $(n=33)$ & $P$ Value \\
\hline \multicolumn{4}{|l|}{ Clinical characteristics } \\
\hline Globulin, mg/dL & $28.18( \pm 7.97)$ & $27.94( \pm 6.59)$ & 0.764 \\
\hline Albumin, mg/dL & $31.76( \pm 5.79)$ & $34.91( \pm 4.13)$ & $<0.01^{\star *}$ \\
\hline 24-h urine protein, $(\mathrm{g} / 24 \mathrm{~h})$ & $1.97( \pm 2.59)$ & $1.88( \pm 3.11)$ & 0.433 \\
\hline C3 Levels, mg/dL & $0.66( \pm 0.32)$ & $0.77( \pm 0.29)$ & 0.143 \\
\hline C4 Levels, mg/dL & $0.12( \pm 0.08)$ & $0.14( \pm 0.06)$ & 0.323 \\
\hline CRP (mg/dL) & $12.36( \pm 14.00)$ & $11.95( \pm 16.98)$ & 0.869 \\
\hline D-dimer (mg/dL) & $1.74( \pm 1.28)$ & $0.75( \pm 0.82)$ & $<0.001^{\star * \star}$ \\
\hline $\mathrm{Cr}(\mu \mathrm{mol} / \mathrm{L})$ & $86.95( \pm 80.42)$ & $66.07( \pm 36.13)$ & 0.200 \\
\hline BUN (mmol/L) & $8.04( \pm 5.66)$ & $7.07( \pm 4.69)$ & 0.491 \\
\hline $\mathrm{UA}(\mu \mathrm{mol} / \mathrm{L})$ & $362.20( \pm 129.73)$ & $339.38( \pm 142.41)$ & 0.523 \\
\hline $\operatorname{GFR}\left(\mathrm{mL} / \mathrm{min} / 1.73 \mathrm{~m}^{2}\right)$ & $117.37( \pm 56.97)$ & $140.31( \pm 83.36)$ & 0.223 \\
\hline ESR (mm/hour) & $50.53( \pm 32.03)$ & $42.59( \pm 28.74)$ & 0.271 \\
\hline PLT $\left(10^{9} / \mathrm{L}\right)$ & $149.32( \pm 79.69)$ & $176.09( \pm 90.84)$ & 0.099 \\
\hline TSH (mlU/L) & $2.78( \pm 2.31)$ & $2.69( \pm 2.21)$ & 0.830 \\
\hline T3 Levels (ng/dL) & $3.20( \pm 1.03)$ & $3.71( \pm 1.06)$ & $<0.05^{\star}$ \\
\hline T4 Levels $(\mu \mathrm{g} / \mathrm{dL})$ & $12.51( \pm 3.43)$ & $14.97( \pm 2.14)$ & $<0.05^{\star}$ \\
\hline \multicolumn{4}{|l|}{ Blood Cells } \\
\hline Erythrocyte, (\%) & $68.66( \pm 118.01)$ & $40.23( \pm 66.60)$ & 0.419 \\
\hline WBC, $\left(10^{9} / \mathrm{L}\right)$ & $4.73( \pm 2.42)$ & $5.43( \pm 3.62)$ & 0.255 \\
\hline CD3+ cells, $(\%)$ & $0.73( \pm 0.50)$ & $0.67( \pm 0.40)$ & 0.605 \\
\hline CD3+CD4+ cells, $(\%)$ & $0.33( \pm 0.22)$ & $0.30( \pm 0.23)$ & 0.591 \\
\hline CD3+CD8+ cells, (\%) & $0.39( \pm 0.29)$ & $0.36( \pm 0.19)$ & 0.563 \\
\hline B cells, (\%) & $0.15( \pm 0.16)$ & $0.10( \pm 0.13)$ & $<0.05^{\star}$ \\
\hline NK cells, $(\%)$ & $0.06( \pm 0.06)$ & $0.06( \pm 0.04)$ & 0.929 \\
\hline \multicolumn{4}{|l|}{ Autoantibodies } \\
\hline Anti-dsDNA antibodies, $\mathrm{U} / \mathrm{mL}$ & $746.12( \pm 441.82)$ & $314.56( \pm 223.99)$ & $<0.05^{\star}$ \\
\hline Anti-Sm antibodies, n (\%) & $10(21.28 \%)$ & $8(24.24 \%)$ & 0.754 \\
\hline
\end{tabular}




\begin{tabular}{|llll|}
\hline Anti-RNP antibodies, $\mathrm{n}(\%)$ & $16(34.04 \%)$ & $13(39.39 \%)$ & 0.624 \\
\hline Anti-SSA antibodies, $\mathrm{n}(\%)$ & $19(40.43 \%)$ & $14(42.42 \%)$ & 0.858 \\
\hline Anti-SSB antibodies, $\mathrm{n}(\%)$ & $4(8.51 \%)$ & $2(6.06 \%)$ & 1.000 \\
\hline Anti-Rib-P, $\mathrm{n}(\%)$ & $10(21.28 \%)$ & $11(33.33 \%)$ & 0.228 \\
\hline Anti- $\beta 2-\mathrm{GPI}, \mathrm{n}(\%)$ & $1(2.13 \%)$ & $0(0 \%)$ & 1.000 \\
\hline $\operatorname{lgM}(\mathrm{mg} / \mathrm{dL})$ & $1.13( \pm 0.72)$ & $0.76( \pm 0.41)$ & $<0.01^{\star \star}$ \\
\hline $\lg \mathrm{(mg} / \mathrm{dL})$ & $14.52( \pm 6.79)$ & $12.56( \pm 5.34)$ & 0.172 \\
\hline $\lg A(\mathrm{mg} / \mathrm{dL})$ & $2.49( \pm 1.60)$ & $2.56( \pm 1.19)$ & 0.857 \\
\hline $\lg \mathrm{m}(\mathrm{mg} / \mathrm{dL})$ & $0.26( \pm 0.44)$ & $0.23( \pm 0.41)$ & 0.620 \\
\hline
\end{tabular}

Data are expressed as number (percentage), or mean \pm standard deviation (SD) values. P values are based on independent sample t-test and Chi-square test for normally distributed variables. ${ }^{*} P<0.05$, $* * P$ $<0.01, \star \star \star P<0.005$, vs. Low RBANS Score. The Graph Pad Prism 7.0 and SPSS Statistics 16.0 software were used for statistical analysis.

\subsection{Correlation analysis between cognitive function and IgG, IgM dsDNA, and albumin levels}

The characteristics of SLE are high levels of autoantibodies and damages to multiple organs [41]. The serum anti-dsDNA antibodies are considered a diagnostic marker and one of the classification criteria for SLE [42-44]. Moreover, abnormal immunoglobulin levels were observed in many of the patients. The results showed that the IgG level was inversely associated with neuropsychological tests of immediate memory (List Learning: $r=-0.344, \mathrm{P}<0.01$; Immediate Story Recall: $r=-0.264, P<0.05$; Fig. $2 \mathrm{~A}$ and 2B). Besides, attention (Digit Span: $r=-0.288, P<0.01$; Coding: $r=-0.294, P<0.05$; Fig. 2C and 2D) and delayed memory (Delayed Story Recall: $r=-0.289, P<0.01$; Figure Recall: $r=-0.275, P<0.05$; Fig. $2 \mathrm{E}$ and $2 \mathrm{~F}$ ) showed a similar correlation, while no significant correlation was found with the other six cognitive functions (Supplementary Fig. 1). The serum IgM level showed a significant negative correlation with Immediate Story Recall $(r=-0.267, P<0.05$; Fig. 2G), Picture Naming $(r=-0.299, P<$ 0.01; Fig. 2H), Digit Span ( $r=-0.243, P<0.05$; Fig. 2l), Delayed Story Recall $(r=-0.299, P<0.01$; Fig. $2 \mathrm{~J})$, and Figure Recall $(r=-0.254, P<0.05 ;$ Fig. $2 \mathrm{~K}$ ). Nevertheless, no correlation was found with other cognitive functions (Supplementary Fig. 2). However, dsDNA did not have a significant correlation with any cognitive function (Supplementary Fig. 3).

The cerebrospinal fluid (CSF)/serum quotient of albumin, known as quotient albumin (Q albumin), is widely accepted as a biomarker for estimating blood-brain barrier (BBB) function [45]; BBB plays a critical role in the pathogenesis of NPSLE [46]. The potential relationship between the serum albumin levels in SLE and cognitive dysfunction was analyzed. The results showed that the albumin levels correlated positively with List Learning, Immediate Story Recall, and Figure Copy (all $P<0.05$; Fig. $2 \mathrm{~L}, \mathrm{M}$, 
and 2N), as well as List Recognition Test and Delayed Story Recall (both $P<0.01$; Fig. 20 and 2P), but had no relationship with other cognitions (Supplementary Fig. 4).

\subsection{Correlation analysis between cognitive function and C3, C4, and lipoprotein levels}

Studies indicated that a significant higher prevalence of thyroid autoantibodies was observed in patients with SLE compared with HCs [47-48]. Many studies tried to associate thyroid abnormalities with clinical findings of SLE, but with no unified conclusion [49-51]. This study explored the relationship between the serum T3 and T4 levels and cognitive functions in patients with SLE. A regression analysis revealed that the serum T3 levels positively correlated with Immediate Memory, including List Learning $(r=0.293, P<$ 0.05; Fig. 3A) and Immediate Story Recall $(r=0.269, P<0.05$; Fig. 3B). Moreover, Figure Copy $(r=0.321, P$ $<0.05$; Fig. 3C), Digit Span ( $r=0.285, P<0.05$; Fig. 3D), List Recall $(r=0.187, P<0.05$; Fig. 3E), List Recognition test $(r=0.245, P<0.01$; Fig. 3F), and Delayed Story Recall $(r=0.258, P<0.05$; Fig. $3 \mathrm{G})$ also correlated positively with the T3 level. The serum T4 level positively correlated with up to nine items $(0.275 \leq r \leq 0.417$; all $P<0.05$; Fig. 3H-3P), including List Learning, Immediate Story Recall, Figure Copy, Digit Span, Coding, List Recall, List Recognition test, Delayed Story Recall, and Figure Recall.

The results indicated that T3 and T4 levels positively correlated with most cognitive functions. Increasing evidence shows that thyroid hormones (mainly T3 and T4) were involved in all aspects of lipid metabolism [52-54]. The relationship between thyroid hormones and lipid metabolism is evident in patients with thyroid dysfunction [55-56]. Moreover, lipid metabolism was markedly altered in patients with SLE [57]. The serum of 81 patients was collected for ELISA, and the expression of four lipoproteins (APOE, APOA1, IGF-1, and IGFBP7) was analyzed to explore any correlation between lipid metabolism and cognitive dysfunction in patients with SLE. The results showed no significant difference in the expression of these four proteins between the two groups (Fig. 4A, 4B, 4C, and 4D). Then, the correlations between the levels of these four lipoproteins and cognitive function were analyzed. The serum APOE levels significantly negatively correlated with Line Orientation ( $r=-0.206, P<0.05$; Fig. 4E), and IGFBP7 and Figure Recall $(r=-0.223, P<0.05$; Fig. 4F), whereas APOA1 showed a positive correlation with Coding $(r$ $=-0.207, P<0.05 ;$ Fig. 4G). Apart from this, no other correlations were found.

\section{Discussion}

In the present study, the cognitive function was lower in patients with SLE compared with HCs. Lower education; higher SLEDAI score; lower albumin, T3, and T4 levels; and higher D-dimer, anti-dsDNA antibodies, and lgM levels were found in patients with low cognition compared with those with high cognition. Besides, cognitive function was closely associated with the $\operatorname{lgG}$, IgM, albumin, T3, and T4 levels in patients. Apart from this, four lipoproteins (APOE, APOA1, IGF-1, and IGFBP7) were detected by ELISA. Although these four lipoproteins showed no difference between the two groups, APOE, APOA1, and IGFBP7 expression correlated with cognition. An important finding of the present study was that the abnormal levels of serum T3, T4, and lipoproteins might be associated with cognitive dysfunction in SLE. 
NPSLE is a severe complication of SLE, which results in severe neurodegenerative changes and threatens life [58]. NPSLE is characterized by a variety of neurological manifestations, making the diagnosis of NPSLE a formidable challenge for rheumatologists [59-60]. Patients with NPSLE had multiple NP events, of which $91.2 \%$ affected the CNS, including cognitive dysfunction, headache, mood disorder, seizures, anxiety, and psychoses. The most common clinical manifestations were cognitive dysfunction, which occurred in $42.1 \%$ of patients [61]. In addition, this study showed that nervous system involvement was the initial presentation of SLE, while more than $50 \%$ of NPSLE occurred within the first 5 years after the onset of SLE [61]. This finding indicated that patients with SLE were likely to undergo cognitive dysfunction before the diagnosis of NPSLE. Therefore, 121 patients with SLE and $65 \mathrm{HCs}$ were recruited for the cognitive assessment using RBANS. The results showed that the cognitive functions of patients with SLE, including immediate memory, visuospatial/constructional, attention, and delayed memory, were significantly lower than the cognitive functions of HCs before the diagnosis of NPSLE. Patients with SLE had a worse response in the lingual gyrus compared with the HCs; this region was associated with visual attention, visual encoding/processing, and working memory [62-63]. This opinion might explain why patients with SLE performed worse on attention, memory task, and visuospatial/constructional memory.

SLE is a prototypic autoimmune disease caused by the loss of B cell tolerance and subsequent recognition of self-antigens and becoming autoreactive [64-65]. In this study, the proportion of B cells obviously increased in patients. Also, the B cell hyper-reactivity could induce an accumulation of autoreactive plasma cells [66], which secreted a variety of autoantibodies, including anti-dsDNA, anti-Sm, anti-Ro, anti-SSA, anti-SSB, anti-Rib-P, and anti- $\beta 2-G P I$, to mediate the occurrence of SLE [67]. The levels of anti-dsDNA and IgM increased in the low-cognition group compared with the high-cognition group, but anti-Sm, anti-RNP, anti-SSA, anti-SSB, anti-Rib-P, anti-B2-GPI, IgG, IgA, and IgE levels all showed no difference. The serum levels of anti-dsDNA antibodies fluctuated with disease activity, whereas others were not altered (anti-Sm, anti-Ro, anti-La, anti-cardiolipin) [68] owing to the different origins of antibodysecreting cells, which partially explained the results. However, in a cohort of 100 randomly selected patients, the higher positive rate of IgG anti-cardiolipin antibodies was found in patients with moderate/severe cognitive impairment, but no difference was found in the incidence of other autoantibodies [67]. Besides, in the present study, the plasma albumin level, as a biomarker of disease activity in SLE [69], decreased in the low-cognition group. The results indicated that the changes in serum $\operatorname{lgM}$, dsDNA, and albumin levels might be associated with cognitive dysfunction in patients with SLE. As expected, IgM showed negative correlations with cognitive function, albumin correlated positively with cognitive function, but dsDNA showed no correlation.

The association between thyroid disease and SLE has been reported for more than 50 years [70]. Both hypothyroidism and thyroid nodules are found more frequently in patients with SLE than in the general population [71]. The rate of thyroid cancer is twice as prevalent in patients with SLE as in those without SLE [72]. In recent years, the impact of serum thyroxine levels on cognitive function has attracted widespread attention gradually, but no consistent conclusions have been drawn. A study on mild cognitive impairment and dementia showed that patients with relatively high T3 levels showed impairment in memory and visuospatial and executive functions [73]. However, patients with acute 
coronary syndrome having low T3 levels had a poorer health-related quality of life (including general health, social functioning, and role emotional) than those with normal levels at a 1-year follow-up [74]. A study on subjective cognitive decline showed that higher T3 levels were associated with better verbal memory performance (immediate and delayed recall tasks) in APOE $\varepsilon 4$ carriers [75]. The results showed that T3 and T4 levels decreased in patients with low cognition, and a significant positive correlation was found between T3 and T4 levels and cognitive function, which was consistent with the aforementioned view and supported that T3 and T4 had protective cognitive functions.

Dyslipidemia is one of the major risk factors for SLE, leading to a high prevalence of premature atherosclerosis and coronary artery disease in patients with SLE [76-77]. In addition, impaired renal function is also associated with dyslipidemia [78-79]. Thyroid hormones influence all aspects of lipid metabolism; especially T3 induces LDL receptor gene expression, enhancing LDL clearance [80]. A report revealed that low free T3 was an independent risk factor for dyslipidemia in patients with SLE [81]. The present study did not show the difference in the expression of serum lipoproteins (APOE, APOA1, IGF-1, and IGFBP7) between the two groups, but three of the aforementioned parameters showed a correlation with the cognitive function: APOE negatively correlated with Line Orientation, APOA1 positively correlated with Coding, and IGFBP7 negatively correlated with Figure Recall. Four years ago, an interesting result was reported: APOE knock-out (KO) mice had synaptic loss and cognitive dysfunction. Although these mice had synaptic loss and dysfunction similar to that in APOE KO mice, they did not have the learning and memory impairment observed in APOE KO mice; also, the memory deficit in the APOE KO mice was specific to female mice [82]. These results indicated that the cognitive impairment in mice was specifically caused by abnormal peripheral blood lipids but not by the expression of APOE in the brain. In $A D$, accelerated cognitive decline and abnormal internal environment, structure, and function of the brain were also found in APOE $\varepsilon 4$ carriers [83]. Leon et al. compiled "The Role of APOE in Cerebrovascular Dysfunction" and pointed out multiple mechanisms of APOE involvement in cognitive dysfunction [84], which could be used to explain the results of the present study. ApoA1 is responsible for transporting cholesterol to the liver. It is a critical component in the formation of HDL. The overexpression of ApoA1 might effectively inhibit the age-related decline in memory and learning ability [85-86]. Moreover, Kawano et al. found that the levels of ApoA1 were strikingly lower in a group of late-onset nonfamilial AD [87]. These studies and the results of the present study both proved a positive correlation between APOA1 and cognitive functions. IGF-1 is an essential neurotrophic factor produced both peripherally and in the brain; adequate levels of serum IGF-1 may be necessary for normal cognitive functioning [88-89]. The serum IGF-1 levels were significantly elevated in patients with SLE and inversely correlated with age [90]. Shankar et al. supported that both high and low levels of IGF-1 might induce poor cognitive function and that optimum levels of IGF-1 might be associated with better cognitive function [91]. Therefore, this explained why the correlation between IGF and cognitive function was not observed in patients with high and low cognitive function.

\section{Conclusion}


In conclusion, the findings indicated that T3 and T4 levels frequently decreased in patients with low cognition, and the cognitive function was associated with T3, T4, APOE, APOA1, and IGFBP7 levels in patients with SLE. The results suggested that T3 and T4 could be used in clinical practice as biomarkers for cognitive dysfunction in SLE.

\section{Abbreviations}

SLE: Systemic lupus erythematosus; NPSLE: Neuropsychiatric SLE; CNS: systemic lupus erythematosus; central nervous system; LDL: low-density lipoprotein; HDL: high-density lipoprotein; NTIS: No thyroidal illness syndrome; T3: triiodothyronine; T4: thyroxine; TSH: thyroid-stimulating hormone; ACR: American College of Rheumatology; SLEDAl: SLE disease activity index; RBANS: Repeatable battery for the assessment of neuropsychological status; MMPI: Minnesota multiphasic personality inventory; CRP: Creactive protein; Cr: Creatine; BUN: blood urea nitrogen; UA: uric acid; GFR: glomerular filtration rate; ESR: erythrocyte sedimentation rate; PLT: platelets; TSH: thyroid stimulating hormone; CSF: cerebrospinal fluid; BBB: Blood-brain barrier; APOE: Apolipoprotein E; APOA1: apolipoprotein A1; IGF-1: insulin-Like growth factor-1; IGFBP7: insulin-like growth factor binding protein 7; AD: Alzheimer's disease.

\section{Declarations}

\section{Ethics approval and consent to participate}

This research was approved by the Ethics Committee at The Affiliated Drum Tower Hospital of Nanjing University Medical School (ID: SC201700201) and was undertaken according to the guidelines of the Declaration of Helsinki. All recruited patients and healthy controls signed informed consent forms.

\section{Consent for publication}

Not applicable.

\section{Availability of data and materials}

The datasets generated during and analyzed during the current study are not publicly available due to respect participants' rights to privacy and to protect their identity, but are available from the corresponding author on reasonable request.

\section{Competing interests}

The authors declare that they have no competing interests.

\section{Funding}

The present work was supported by the Natural Science Foundation of China (32070883, 31872732 and 81871319), Jiangsu Province Six Talent Peaks Project (2016-WSN-148 and YY-021), Jiangsu Province 
key Social and Development R\&A Grant (BE2019706 and BE2019617), Jiangsu Provincial Medical Youth Talent (QNRC2016005) and Research Project of Jiangsu Province Health Committee (H2019060).

\section{Authors' contributions}

Huan Dou, Yayi Hou and Jun Liang conceived and designed the project. Li Lu, Jinglei Chen and Wei Kong performed the experiments, analyzed the data. Huan Dou, Kangxing Zhou and Jun Liang interpreted the patient data. Li Lu, wrote the manuscript, and Huan Dou and Jun Liang revised the manuscript. All authors read and approved the final manuscript.

\section{Acknowledgements}

Not applicable.

\section{References}

1. Tsokos GC, Lo MS, Costa Reis P, Sullivan KE. New insights into the immunopathogenesis of systemic lupus erythematosus. Nat Rev Rheumatol. 2016 Nov 22; 12(12):716-730.

2. Gulinello M, Wen J, Putterman C. Neuropsychiatric symptoms in lupus. Psychiatr Ann. (2012) 42:322-8. 10.3928/00485713-20120906-05

3. Jarpa E, Babul M, Calderón J, González M, Martínez ME, Bravo-Zehnder M, Henríquez C, Jacobelli S, González A, Massardo L. Common mental disorders and psychological distress in systemic lupus erythematosus are not associated with disease activity.Lupus. 2011 Jan; 20(1):58-66.

4. Unterman A, Nolte JE, Boaz M, Abady M, Shoenfeld Y, Zandman-Goddard G. Neuropsychiatric syndromes in systemic lupus erythematosus: a meta-analysis.Semin Arthritis Rheum. 2011 Aug; 41(1):1-11.

5. Borowoy AM, Pope JE, Silverman E, Fortin PR, Pineau C, Smith CD, Arbillaga H, Gladman D, Urowitz M, Zummer M, Hudson M, Tucker L, Peschken C. Neuropsychiatric lupus: the prevalence and autoantibody associations depend on the definition: results from the 1000 faces of lupus cohort. Semin Arthritis Rheum. 2012 Oct; 42(2):179-85.

6. Chen Chen, Linyu Geng, Xue Xu, Wei Kong, Yayi Hou, Genhong Yao, Xuebing Feng, Huayong Zhang, Jun Liang. Comparative proteomics analysis of plasma protein in patients with neuropsychiatric systemic lupus erythematosus.

7. Hanly JG. Diagnosis and management of neuropsychiatric SLE. Nat Rev Rheumatol. 2014 Jun; 10(6):338-47.

8. Govoni M, Bortoluzzi A, Padovan M, Silvagni E, Borrelli M, Donelli F, Ceruti S, Trotta F. The diagnosis and clinical management of the neuropsychiatric manifestations of lupus. J Autoimmun. 2016 Nov; 740):41-72.

9. Angelina R Sutin, Yannick Stephan, Martina Luchetti, Antonio Terracciano, et al. Five-factor model personality traits and cognitive function in five domains in older adulthood. BMC Geriatr. 2019 Dec 
5;19(1):343.

10. Y.I. Shyu, P.K. Yip Factor structure and explanatory variables of the Mini-Mental State Examination (MMSE) for elderly persons in Taiwan J. Formos. Med. Assoc.100 (10) (2001), pp. 676-683.

11. Z.S. Nasreddine, N.A. Phillips, V. Bedirian, et al. The Montreal Cognitive Assessment, MoCA: a brief screening tool for mild cognitive impairment J. Am. Geriatr. Soc., 53 (4) (2005), pp. 695-699.

12. J.E. Galvin, C.M. Roe, K.K. Powlishta, et al. The AD8: a brief informant interview to detect dementia Neurology, 65 (4) (2005), pp. 559-564.

13. H. Li, T. Zhang, T.C. Yu, C.C. Lin, A.M.K. Wong, Combine wireless sensor network and multimedia technologies for cognitive function assessment, in: Proceedings of the Third International Conference on Intelligent Control and Information Processing (ICICIP), 2012, pp. 717-720.

14. William F Goette, Andrew L Schmitt. Examination of Regression-based Discrepancy Scores for the RBANS in Detecting Cognitive Impairment from an Archival Sample. Arch Clin Neuropsychol. 2019 Nov 27;34(8):1329-1339.

15. Andrea Gogos, Nicole Joshua, Susan L Rossell. Use of the Repeatable Battery for the Assessment of Neuropsychological Status (RBANS) to investigate group and gender differences in schizophrenia and bipolar disorder. Aust N Z J Psychiatry. 2010 Mar;44(3):220-9.

16. William W Beatty. RBANS analysis of verbal memory in multiple sclerosis. Arch Clin Neuropsychol. 2004 Sep;19(6):825-34.

17. Fiorenzo Gaita, Laura Corsinovi, Matteo Anselmino, et al. Prevalence of silent cerebral ischemia in paroxysmal and persistent atrial fibrillation and correlation with cognitive function. J Am Coll Cardiol. 2013 Nov 19;62(21):1990-1997.

18. Robert J Quon, Morgan T Mazanec, Samantha S Schmidt. Antiepileptic drug effects on subjective and objective cognition. Epilepsy Behav. 2020 Mar;104(Pt A):106906.

19. Amaya-Amaya J, Sarmiento-Monroy JC, Caro-Moreno J, Molano-Gonzalez N, Mantilla RD, RojasVillarraga A, et al. Cardiovascular disease in latin american patients with systemic lupus erythematosus: a cross-sectional study and a systematic review. Autoimmune Dis. 2013; 2013: 7943

20. Borba EF, Bonfá E. Dyslipoproteinemias in systemic lupus erythematosus: influence of disease, activity, and anticardiolipin antibodies. Lupus. 1997; 6:533-539.

21. Tisseverasinghe A, Lim S, Greenwood C, Urowitz M, Gladman D, Fortin PR. Association between serum total cholesterol level and renal outcome in systemic lupus erythematosus. Arthritis Rheum. 2006; 54:2211-2219.

22. S.A. Karimi, I. Salehi, A. Komaki, et al. Effect of high-fat diet and antioxidants on hippocampal longterm potentiation in rats: an in vivo study. Brain Res.1539 (2013), pp. 1-6.

23. A. Lindqvist, P. Mohapel, B. Bouter, et al. High-fat diet impairs hippocampal neurogenesis in male rats. Eur. J. Neurol.13 (2006), pp. 1385-1388.

24. A. Wu, R. Molteni, Z. Ying, F. Gomez-Pinilla. A saturated-fat diet aggravates the outcome of traumatic brain injury on hippocampal plasticity and cognitive function by reducing brain-derived neurotrophic 
factor. Neuroscience, 119 (2003), pp. 365-375.

25. Neto AM, Zantut-Wittmann DE. Abnormalities of Thyroid Hormone Metabolism during Systemic Illness: The Low T3 Syndrome in Different Clinical Settings. International Journal of Endocrinology. 2016; 2016: 2157583

26. Watad A, Mahroum N, Whitby A, Gertel S, Comaneshter D, Cohen AD, et al. Hypothyroidism among SLE patients: Case-control study. Autoimmun Rev. 2016; 15: 484-486.

27. Pingitore A, Galli E, Barison A, lervasi A, Scarlattini M, Nucci D, et al. Acute effects of triiodothyronine (T3) replacement therapy in patients with chronic heart failure and low-T3 syndrome: a randomized, placebo-controlled study. Journal of Clinical Endocrinology \& Metabolism. 2008; 93: 1351-1358.

28. X. Zhu and S. Y. Cheng, "New insights into regulation of lipid metabolism by thyroid hormone," Current Opinion in Endocrinology, Diabetes and Obesity, vol. 17, no. 5, pp. 408-413, 2010.

29. R. Asher. Myxoedematous madness. Br Med J, 2 (4627) (1949), pp. 555-562.

30. T.W. Heinrich, G. Grahm Hypothyroidism presenting as psychosis: myxedema madness revisited Prim Care Companion J Clin Psychiatry, 5 (6) (2003), pp. 260-266.

31. C.D. Smith, R. Grondin, W. LeMaster, et al. Reversible cognitive, motor, and driving impairments in severe hypothyroidism. Thyroid, 25 (1) (2015), pp. 28-36.

32. Smith EL, Shmerling RH. The American College of Rheumatology criteria for the classification of systemic lupus erythematosus: Strengths, weaknesses, and opportunities for improvement. Lupus 1999;8:586-95.

33. Gladman DD, Ibanez D, Urowitz MB. Systemic lupus erythematosus disease activity index 2000. J Rheumatol 2002;29:288-91.

34. Hanly JG. ACR classification criteria for systemic lupus erythematosus: Limitations and revisions to neuropsychiatric variables. Lupus 2004;13:861-4.

35. Hay EM, Black D, Huddy A, Creed F, Tomenson B, et al. Psychiatric disorder and cognitive impairment in systemic lupus erythematosus. Arthritis Rheum. 1992;35:411-6.

36. Kozora E, Thompson LL, West SG, Kotzin BL. Analysis of cognitive and psychological deficits in systemic lupus erythematosus patients without overt central nervous system disease. Arthritis Rheum. 1996;39:2035-45.

37. Hahn RC, Petti DB. Minnesota multiphasic personality inventory-rated depression and the incidence of breast cancer. Cancer. 1988;61:845.

38. Christopher Randolph, Michael C. Tierney, Erich Mohr \& Thomas N. Chase. The Repeatable Battery for the Assessment of Neuropsychological Status (RBANS): Preliminary Clinical Validity. J Clin Exp Neuropsychol. 1998 Jun;20(3):310-9.

39. Cheng Yan, Li Chunbo, Wu Wenyuan. Application evaluation of a repeatable set of neuropsychological status tests. Chin J Psychiatry. February 2010, Vol. 43, No. 1 (in Chinese)

40. Qin Huang, Shuqun Shen, Hang Qu, et al. Expression of HMGB1 and TLR4 in neuropsychiatric systemic lupus erythematosus patients with seizure disorders. Ann Transl Med. 2020 Jan;8(1):9. 
41. Zardi EM, Giorgi C, Zardi DM. Diagnostic approach to neuropsychiatric lupus erythematosus: what should we do? Postgrad Med. 2018;130(6):536-547.

42. Petri M., Orbai A.M., Alarcon G.S., Gordon C., Merrill J.T., Fortin P.R., Bruce I.N., Isenberg D., Wallace D.J., Nived O., et al. Derivation and validation of the systemic lupus international collaborating clinics classification criteria for systemic lupus erythematosus. Arthritis Rheum. 2012;64:2677-2686.

43. Gronwall C., Akhter E., Oh C., Burlingame R.W., Petri M., Silverman G.J. IgM autoantibodies to distinct apoptosis-associated antigens correlate with protection from cardiovascular events and renal disease in patients with SLE. Clin. Immunol. 2012;142:390-398.

44. Barbara Dema, Nicolas Charles. Autoantibodies in SLE: Specificities, Isotypes and Receptors. Antibodies (Basel). 2016 Mar; 5(1): 2.

45. Tibbling G, Link H, Ohman S. Principles of albumin and IgG analyses in neurological disorders. I. Establishment of reference values. Scand J Clin Lab Invest. 1977;37(5):385-90.

46. Abbott NJ, Mendonca LL, Dolman DE. The blood-brain barrier in systemic lupus erythematosus. Lupus. 2003;12(12):908-15.

47. Weetman AP, Walport MJ. The association of autoimmune thyroiditis with systemic lupus erythematosus. Br J Rheumatol (1987) 26:359-61.

48. Miller FW, Moore GF, Weintraub BD, Steinberg AD. Prevalence of thyroid disease and abnormal thyroid function test results in patients with systemic lupus erythematosus. Arthritis Rheum. 1987 Oct; 30(10):1124-31.

49. Magaro M, Zoli A, Altomonte L, Mirone L, La Sala L, Barini A, Scuderi F. The association of silent thyroiditis with active systemic lupus erythematosus. Clin Exp Rheumatol. 1992 Jan-Feb; 10(1):6770.

50. Appenzeller S, Pallone AT, Natalin RA, Costallat LT. Prevalence of thyroid dysfunction in systemic lupus erythematosus. J Clin Rheumatol (2009) 15:117-9.

51. Kumar K, Kole AK, Karmakar PS, Ghosh A. The spectrum of thyroid disorders in systemic lupus erythematosus. Rheumatol Int (2012) 32:73-8.

52. Berti JA, Amaral ME, Boschero AC, Nunes VS, Harada LM, Castilho LN, Oliveira HC. Thyroid hormone increases plasma cholesteryl ester transfer protein activity and plasma high-density lipoprotein removal rate in transgenic mice. Metabolism. 2001 May; 50(5):530-6.

53. Prieur X, Huby T, Coste H, Schaap FG, Chapman MJ, Rodríguez JC. Thyroid hormone regulates the hypotriglyceridemic gene APOA5. J Biol Chem. 2005 Jul 29; 280(30):27533-43.

54. Kok-Yong Chin, Soelaiman Ima-Nirwana, Isa Naina Mohamed, Amilia Aminuddin, Mohamad Hanapi Johari, and Wan Zurinah Wan Ngah. The Relationships between Thyroid Hormones and Thyroidstimulating Hormone with Lipid Profile in Euthyroid Men. Int J Med Sci. 2014; 11(4): 349-355.

55. Rizos CV, Elisaf MS, Liberopoulos EN. Effects of thyroid dysfunction on lipid profile. Open Cardiovasc Med J. 2011; (5):76-84. 
56. Peppa M, Betsi G, Dimitriadis G. Lipid abnormalities and cardiometabolic risk in patients with overt and subclinical thyroid disease. J Lipids. 2011; (2011):575840.

57. Borba EF, Bonfá E. Dyslipoproteinemias in systemic lupus erythematosus: influence of disease, activity, and anticardiolipin antibodies. Lupus. 1997; 6(6):533-9.

58. Dias-Santos A, Proenca RP, Tavares Ferreira J, et al. The role of ophthalmic imaging in central nervous system degeneration in systemic lupus erythematosus. Autoimmun Rev 2018;17:617-24.

59. Aranow C, Diamond B, Backay M. Pathogensesis of the Nervous System. In: Wallace DJ, Hahn BH, editors. Dubois' Lupus Erythematosus and Related Syndromes. Philadelphia: Saunders. 2013:363-7.

60. Ward MM. Premature morbidity from cardiovascular and cerebrovascular diseases in women with systemic lupus erythematosus. Arthritis Rheum. 1999 Feb; 42(2):338-46.

61. Yongwen Zhang, Huanhuan Han, Lanfang Chu. Neuropsychiatric Lupus Erythematosus: Future Directions and Challenges; a Systematic Review and Survey. Clinics (Sao Paulo). 2020; 75: e1515.

62. Benedek M, Jauk E, Beaty RE, Fink A, Koschutnig K, Neubauer AC. Brain mechanisms associated with internally directed attention and self-generated thought. Sci Rep. 2016 Mar 10; 60:22959.

63. Mattfeld AT, Whitfield-Gabrieli S, Biederman J, et al. Dissociation of working memory impairments and attention-deficit/hyperactivity disorder in the brain. Neuroimage Clin 2016;10:274-82.

64. Giltiay, N.V.; Chappell, C.P.; Clark, E.A. B-cell selection and the development of autoantibodies. Arthritis Res.Ther. 2012, 14 (Suppl. 4), S1.

65. Han, S.; Zhuang, H.; Shumyak, S.; Yang, L.; Reeves, W.H. Mechanisms of autoantibody production insystemic lupus erythematosus. Front. Immunol. 2015, 6, 228.

66. Lamagna, C.; Hu, Y.; DeFranco, A.L.; Lowell, C.A. B cell-specific loss of lyn kinase leads to autoimmunity. J. Immunol. 2014, 192, 919-928.

67. Alı 'Duarte-Garcı 'a, Juanita Romero-Dı 'az, Sandra Jua'rez, Alba Cicero-Casarrubias, Hilda FragosoLoyo, Carlos Nu'ñez-Alvarez, Luis Llorente, Jorge Sa'nchez-Guerrero. Disease activity, autoantibodies, and inflammatory molecules in serum and cerebrospinal fluid of patients with Systemic Lupus Erythematosus and Cognitive Dysfunction. PLoS One. 2018; 13(5): e0196487.

68. Liu, Z.; Zou, Y.; Davidson, A. Plasma cells in systemic lupus erythematosus: The long and short of it all. Eur. J. Immunol. 2011, 41, 588-591.

69. Helena Idborg, Susanna Eketjäll, Susanne Pettersson, Johanna T Gustafsson, Agneta Zickert, Marika Kvarnström, Vilija Oke, Per-Johan Jakobsson, Iva Gunnarsson, and Elisabet Svenungsson. TNF-a and plasma albumin as biomarkers of disease activity in systemic lupus erythematosus. Lupus Sci Med. 2018; 5(1): e000260.

70. White RG, Bass BH, Williams E. Lymphadenoid goitre and the syndrome of systemic lupus erythematosus. Lancet 1961;277:368-73.

71. Miller FW, Moore GF, Weintraub BD, Steinberg AD. Prevalence of thyroid disease and abnormal thyroid function test results in patients with systemic lupus erythematosus. Arthritis Rheum 1987;30:1124-31. 
72. Blich M, Rozin A, Edoute Y. Systemic lupus erythematosus and thyroid disease. Isr Med Assoc J 2004;6:218-20.

73. Patrick Quinlan, Arto Nordlund, Karin Lind, Deborah Gustafson, Åke Edman, Anders Wallin. Thyroid Hormones Are Associated with Poorer Cognition in Mild Cognitive Impairment. Dement Geriatr Cogn Disord 2010;30:205-211.

74. Chao Xue, Ling Bian, Yu Shui Xie, Zhao Fang Yin, Zuo Jun Xu, Qi Zhi Chen, Hui Li Zhang, Yu Qi Fan, Run Du, and Chang Qian Wang. Low fT3 is associated with diminished health-related quality of life in patients with acute coronary syndrome treated with drug-eluting stent: a longitudinal observational study. Oncotarget. 2017 Nov 7; 8(55): 94580-94590.

75. Jin San Lee, Yunsoo Soh, Hyug-Gi Kim, Kyung Mi Lee, Young Nam Kwon, Sung Sang Yoon, KeyChung Park, and Hak Young Rhee. Interactive Effects of Apolipoprotein E $\varepsilon 4$ and Triiodothyronine on Memory Performance in Patients With Subjective Cognitive Decline. Front Med (Lausanne). 2020; 7: 298.

76. 76.

77. Manzi S, Meilahn EN, Rairie JE, Conte CG, Medsger TA, Jansenmcwilliams L, et al. Age-specific Incidence Rates of Myocardial Infarction and Angina in Women with Systemic Lupus Erythematosus: Comparison with the Framingham Study. American Journal of Epidemiology. 1997; 145: 408-415.

78. Vaziri ND, Moradi H. Mechanisms of dyslipidemia of chronic renal failure. Hemodialysis International. 2006; 10:1-7.

79. Attman PO, Alaupovic P. Lipid and apolipoprotein profiles of uremic dyslipoproteinemia - relation to renal function and dialysis. Nephron. 1991; 57:401-410.

80. Melpomeni Peppa, GrigoriaBetsi, andGeorge Dimitriadis. Lipid Abnormalities and Cardiometabolic Risk inPatients with Overt and Subclinical Thyroid Disease. Journal of Lipids Volume 2011, Article ID 575840, 9 pages.

81. Xin Zhang, Lirong Liu, Xiaolei Ma, Wei Hu, Xue Xu, Saisai Huang, Bingzhu Hua, Hong Wang, Zhiyong Chen, Lingyun Sun. Clinical significance of non-thyroidal illness syndrome on disease activity and dyslipidemia in patients with SLE. PLoS One. 2020; 15(4): e0231622.

82. Courtney Lane-Donovan, Wen Mai Wong, Murat S Durakoglugil, Catherine R Wasser, Shan Jiang, Xunde Xian, Joachim Herz. Genetic Restoration of Plasma ApoE Improves Cognition and Partially Restores Synaptic Defects in ApoE-Deficient Mice. J Neurosci. 2016 Sep 28; 36(39): 10141-10150.

83. Jialing Fan, Wuhai Tao, Xin Li, He Li, Junying Zhang, Dongfeng Wei, Yaojing Chen, and Zhanjun Zhang. The Contribution of Genetic Factors to Cognitive Impairment and Dementia: Apolipoprotein E Gene, Gene Interactions, and Polygenic Risk. Int J Mol Sci. 2019 Mar; 20(5): 1177.

84. Leon M Tai, Riya Thomas, Felecia M Marottoli, Kevin P Koster, Takahisa Kanekiyo, Alan WJ Morris, and Guojun Bu. The Role of APOE in Cerebrovascular Dysfunction. Acta Neuropathol. 2016 May; 131(5): 709-723.

85. Millán J., Pintó X., Muñoz A., et al. Lipoprotein ratios: physiological significance and clinical usefulness in cardiovascular prevention. Vascular Health and Risk Management. 2009;5:757-765. 
86. Lewis T. L., Cao D., Lu H., et al. Overexpression of human apolipoprotein A-I preserves cognitive function and attenuates neuroinflammation and cerebral amyloid angiopathy in a mouse model of Alzheimer disease. The Journal of Biological Chemistry. 2010;285(47):36958-36968.

87. Kawano M., Kawakami M., Otsuka M., Yashima H., Yaginuma T., Ueki A. Marked decrease of plasma apolipoprotein Al and All in Japanese patients with late-onset non-familial Alzheimer's disease. Clinica Chimica Acta. 1995;239(2):209-211. doi: 10.1016/0009-8981(95)06115-t.

88. Morley JE, Kaiser F, Raum WJ, Perry HM 3rd, Flood JF, Jensen J, et al. Potentially predictive and manipulable blood serum correlates of aging in the healthy human male: progressive decreases in bioavailable testosterone, dehydroepiandrosterone sulfate, and the ratio of insulin-like growth factor 1 to growth hormone. Proc Natl Acad Sci U S A. 1997;94: 7537-7542.

89. Rollero A, Murialdo G, Fonzi S, Garrone S, Gianelli MV, Gazzerro E, et al. Relationship between cognitive function, growth hormone and insulin-like growth factor I plasma levels in aged subjects. Neuropsychobiology. 1998;38: 73-79.

90. Charles W Denko and Charles J Malemud. Age-related changes in Serum Growth Hormone, Insulinlike Growth Factor-1 and Somatostatin in System Lupus Erythematosus. BMC Musculoskelet Disord. 2004; 5: 37.

91. Shankar Tumati, Huibert Burger, Sander Martens, Yvonne T. van der Schouw, and André Aleman. Association between Cognition and Serum Insulin-Like Growth Factor-1 in Middle-Aged \& Older Men: An 8 Year Follow-Up Study. PLoS One. 2016; 11(4): e0154450.

\section{Figures}


A

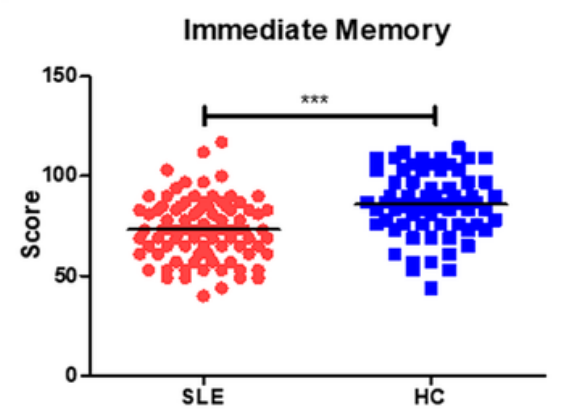

D

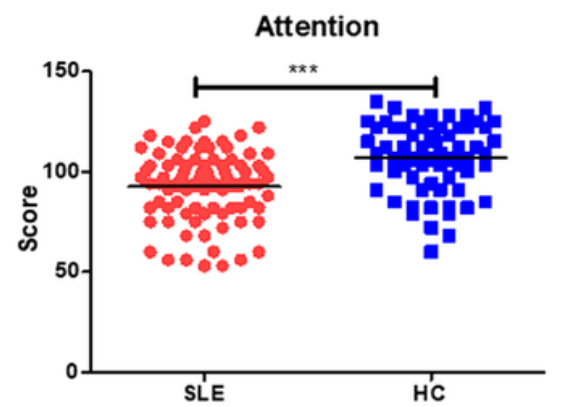

B

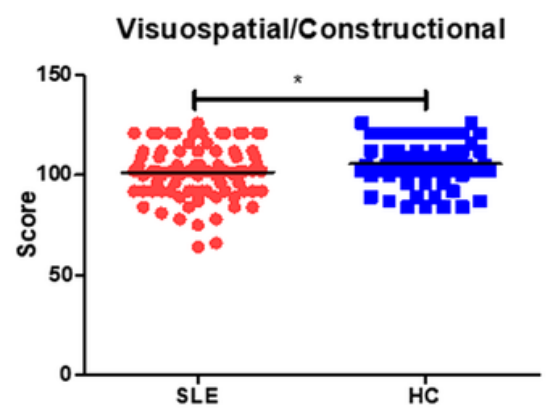

E

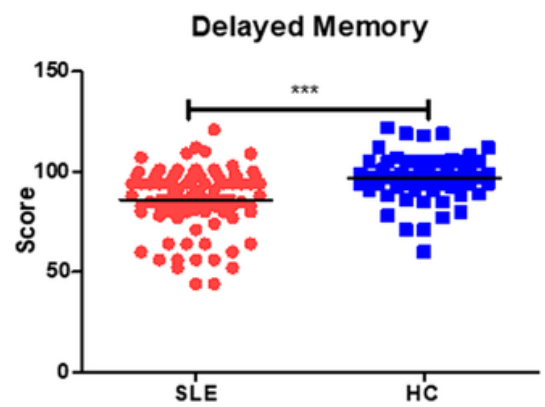

C

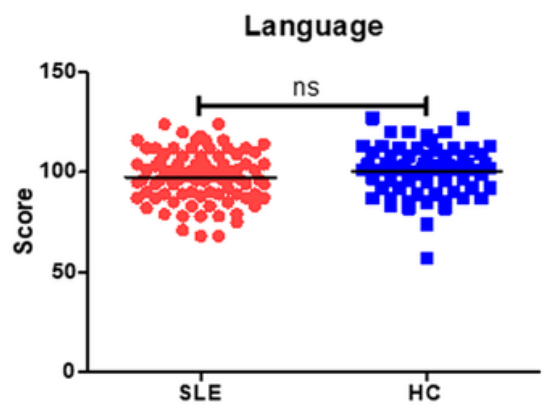

$\mathbf{F}$

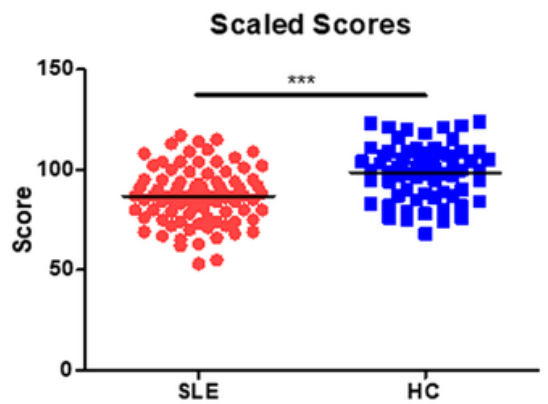

Figure 1

Cognitive function of patients with SLE and HCs The difference of Immediate Memory (A), Visuospatial/Constructional (B), Language (C), Attention (D), Delayed memory (E) between patients with SLE and HCs. Then, scores in the above 5 cognitive areas were converted into scaled scores (F). 
A

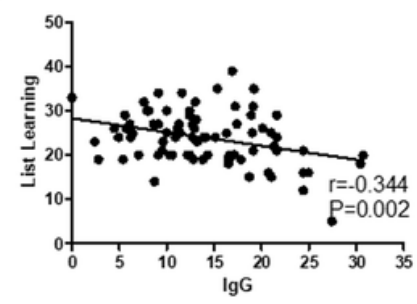

E

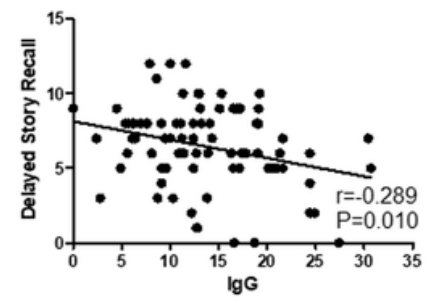

I

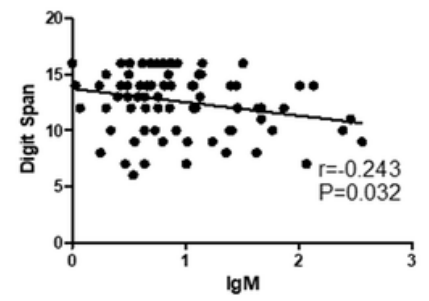

M

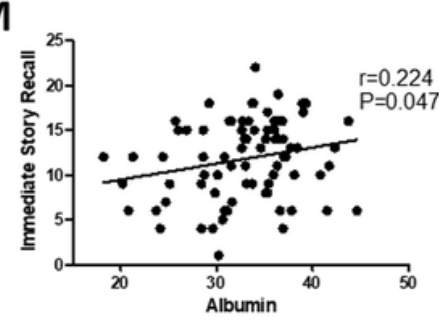

B

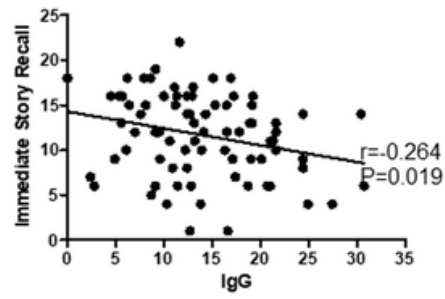

F

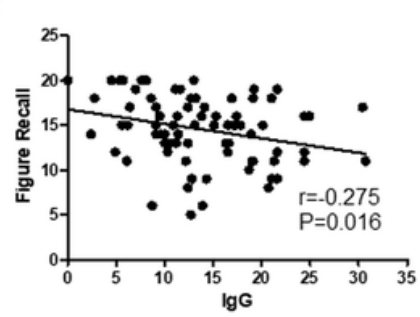

J

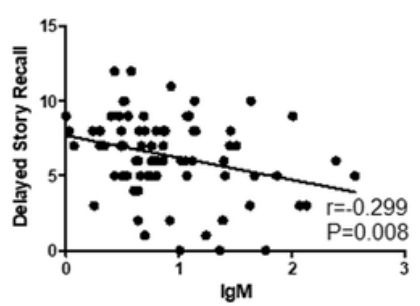

N

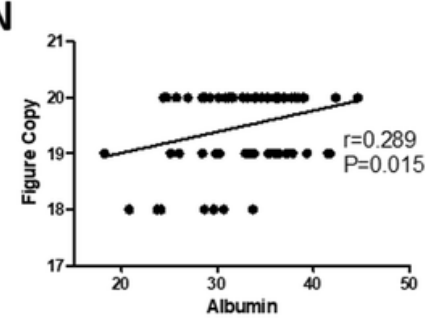

C

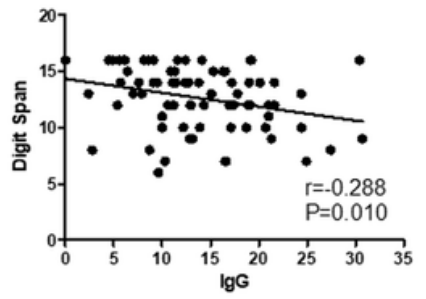

G

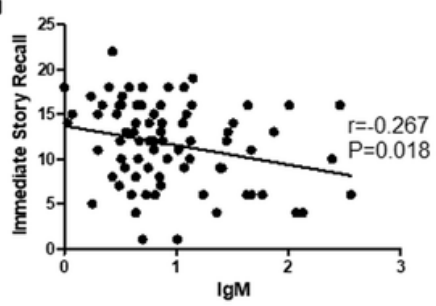

K

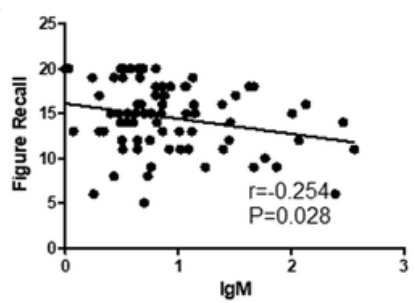

0

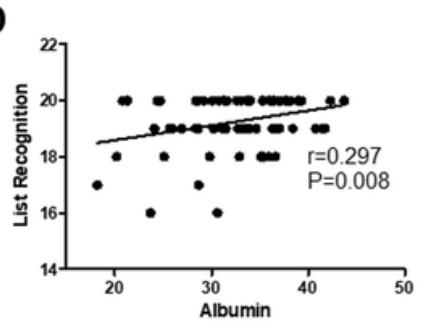

D

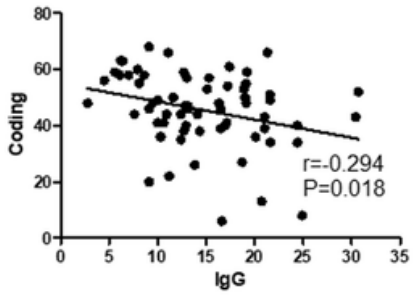

H

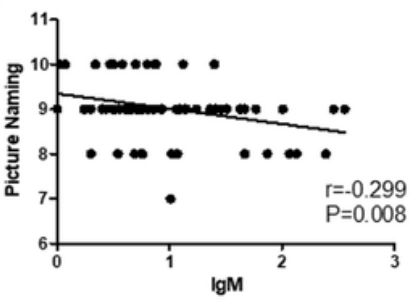

L
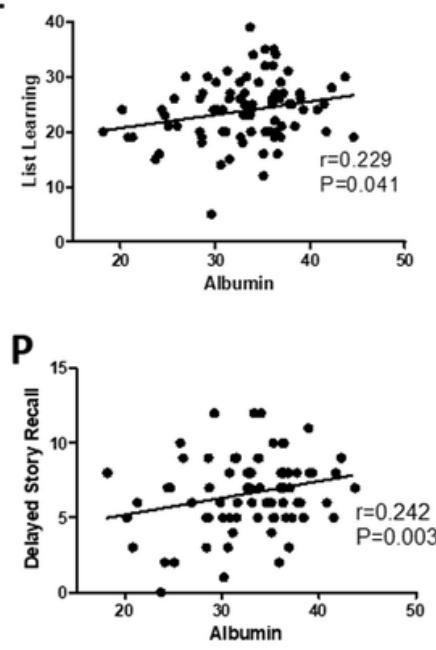

Figure 2

Correlation analysis between cognitive function and $\lg G$, IgM, and albumin levels Correlation between IgG and List learning (A), Immediate Story Recall (B), Digit Span (C), Coding(D), Delayed Story Recall (E), and Figure Recall (F) in patients with SLE. Correlation between IgM and Immediate Story Recall (G), Picture Naming $(\mathrm{H})$, Digit Span (I), Delayed Story Recall $(\mathrm{J})$, and Figure Recall $(\mathrm{K})$ in patients with SLE. Correlation between IgM and List learning (L), Immediate Story Recall (M), Figure Copy $(N)$, List Recognition $(0)$ and Delayed Story Recall $(P)$ in patients with SLE. 
A

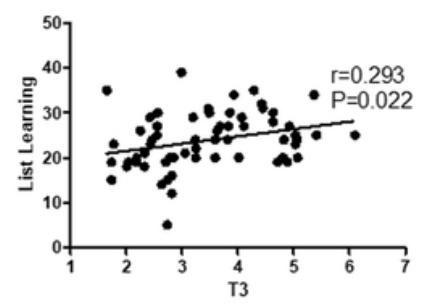

E

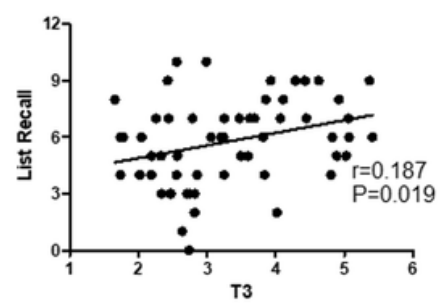

I

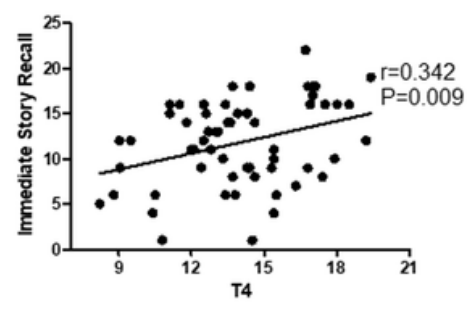

M

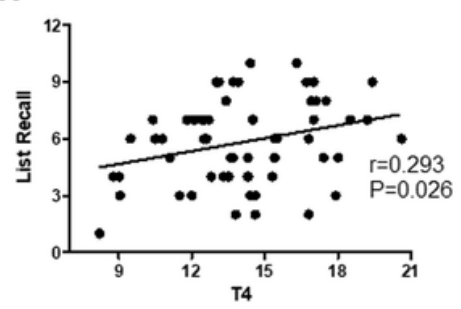

B

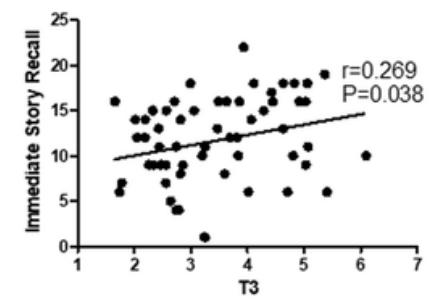

F

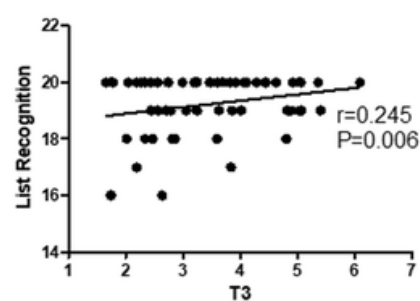

J

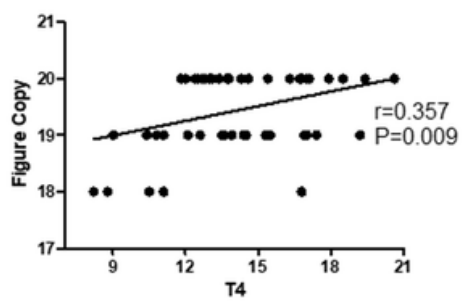

N

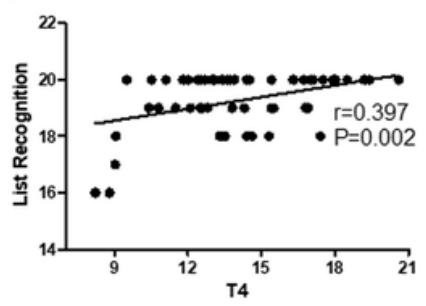

C

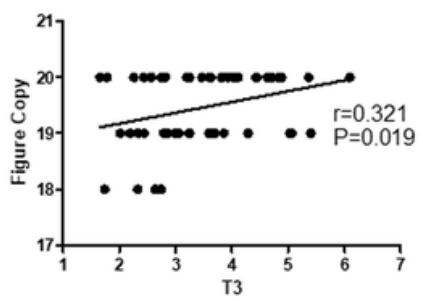

G

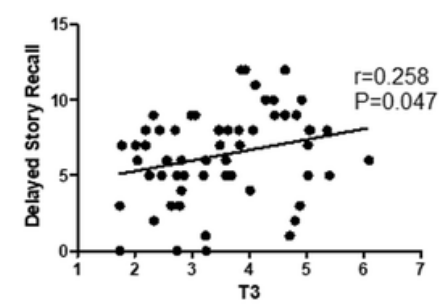

K

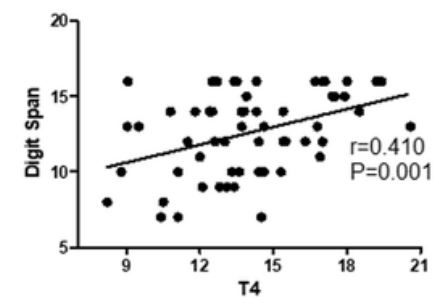

0

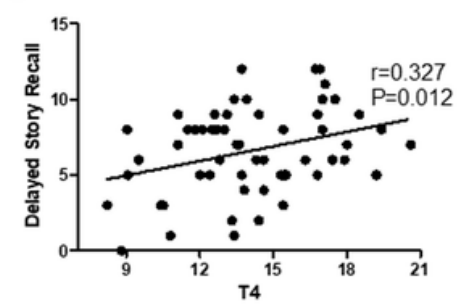

D

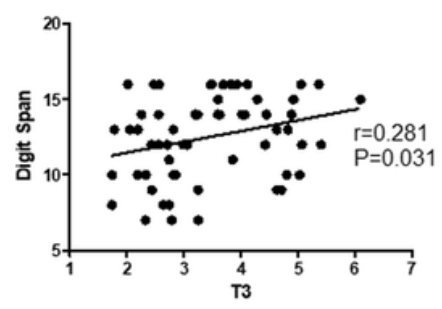

H

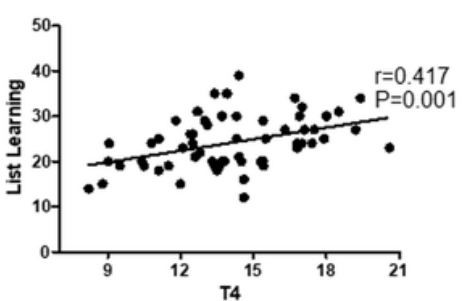

L

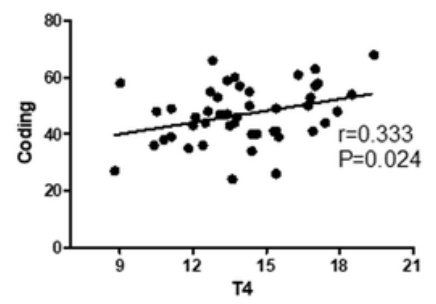

P

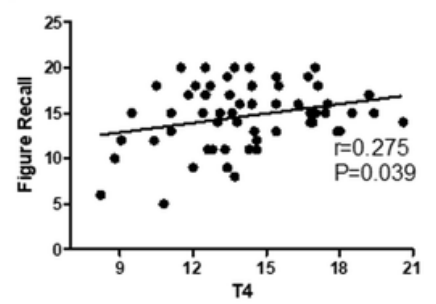

Figure 3

Correlation analysis between cognitive function and T3, T4 serum levels Correlation between T3 and List learning (A), Immediate Story Recall (B), Figure Copy (C), Digit Span (D), List Recall (E), List Recognition $(F)$, and Delayed Story Recall $(G)$ in patients with SLE. Correlation between T4 and List learning $(H)$, Immediate Story Recall (I), Figure Copy (J), Digit Span (K), Coding (L), List Recall (M), List Recognition $(\mathrm{N})$, Delayed Story Recall $(\mathrm{O})$, and Figure Recall $(\mathrm{P})$ in patients with SLE. 

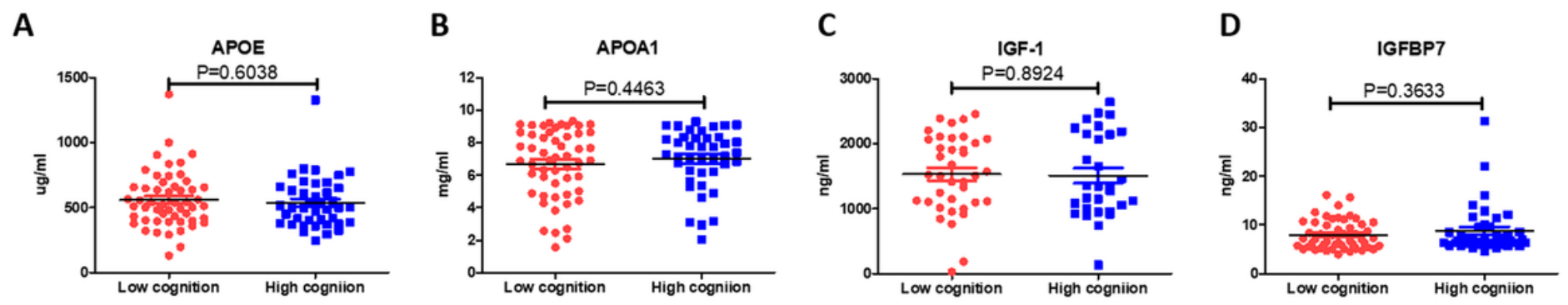

E

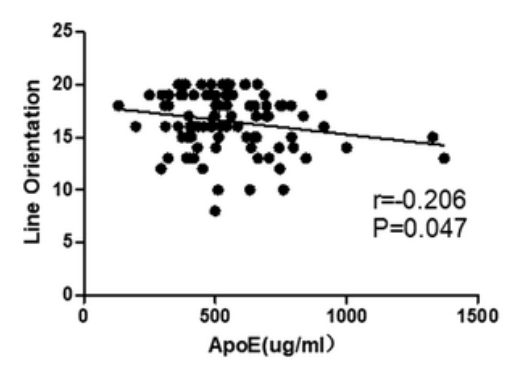

$\mathbf{F}$

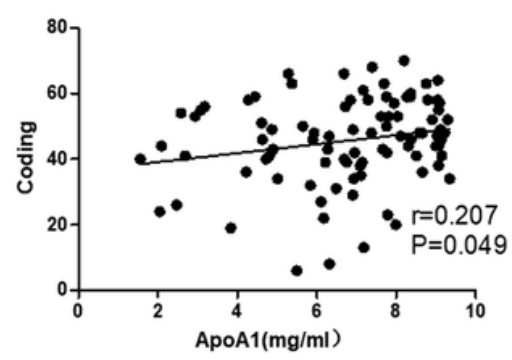

G

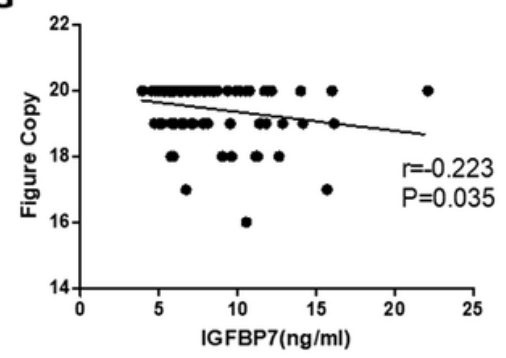

Figure 4

The expression of serum lipoprotein and the correlation between cognitive function The difference of serum APOE (A), APOA1 (B), IGF-1 (C), and IGFBP7 between different cognitive patients with SLE.

Correlation between APOE and List Orientation (E) in patients with SLE. Correlation between APOA1 and Coding (F) in patients with SLE. Correlation between IGFBP7 and Figure Copy (G) in patients with SLE.

\section{Supplementary Files}

This is a list of supplementary files associated with this preprint. Click to download.

- Supplementarymaterials.docx

- Figure2P.tif

- CorrelationanalysisbetweencognitivefunctionandlgGlgMdsDNAalbuminT3T4.docx 